\title{
DSC as a screening tool for rapid co-crystal detection in binary mixtures of benzodiazepines with co-formers
}

\author{
Patrycja Saganowska $^{1}$ - Marek Wesolowski ${ }^{1}$
}

Received: 9 August 2017 / Accepted: 26 November 2017 / Published online: 4 December 2017

(c) The Author(s) 2017. This article is an open access publication

\begin{abstract}
In recent years, co-crystals have been the subject of increasing interest within the pharmaceutical industry because these new solid forms of active pharmaceutical ingredients have the ability to enhance the bioavailability of poorly water-soluble drugs with a low dissolution rate. For this reason, it is crucial to prepare co-crystals of benzodiazepines, i.e. psychoactive drugs with a wide range of medical applications but classified as very slightly or practically insoluble in water. Thus, the objective of this research was to show to what extent the DSC method can be used as a screening tool for detection of cocrystal formation in binary physical mixtures of drugs and co-formers. To obtain potential co-crystals, eight benzodiazepines (diazepam, temazepam, oxazepam, lormetazepam, lorazepam, clonazepam, estazolam and chlordiazepoxide) were gently mixed in an agate mortar at 1:1 molar ratios with nine co-formers-succinic, glutaric, fumaric, citric and $p$ aminobenzoic acids, nicotinamide, saccharin, urea and caffeine, and heated under DSC conditions. A detailed comparison of the DSC scans of mixtures against scans of both ingredients in isolation indicates the occurrence of subtle physical changes in the samples. Thus, additional endothermic or exothermic peaks due to melting or crystallisation suggested the formation of potential co-crystals. To conclude, this study confirms that the DSC method can be used as a rapid screening tool for co-crystal detection. In this case, based on the DSC scans, 15 physical mixtures of benzodiazepines (clonazepam, diazepam, lorazepam, oxazepam and temazepam) with co-formers (citric, fumaric, glutaric, $p$-aminobenzoic and succinic acids, nicotinamide, saccharin and urea) have been selected as potential co-crystals for further detailed study.
\end{abstract}

Keywords Co-crystals $\cdot$ Benzodiazepines $\cdot$ Co-formers $\cdot$ DSC $\cdot$ Screening method

\section{Introduction}

Pharmaceutical researchers have recently being devoting more and more attention to co-crystals. This interest in cocrystals is connected with the possibility of their use as a new solid form of active pharmaceutical ingredients (APIs) [1-4]. Co-crystals are defined as a connection of at least two molecules in a solid state within the same crystal lattice. In relation to pharmaceutical co-crystals, one or even two components are drugs, as opposed to the majority of cases where the second component consists of a neutral molecule or "co-former", which should have a harmless effect on the human body [5, 6]. Non-ionisable or ionisable

Marek Wesolowski

marwes@gumed.edu.pl

1 Department of Analytical Chemistry, Medical University of Gdansk, Gen. J. Hallera 107, 80-416 Gdańsk, Poland (only weakly acidic or basic) compounds are permitted as part of the co-crystallisation process [5]. The co-crystal components are bonded together via non-covalent bonds, predominantly via hydrogen bonding, which explains why no proton transfer occurs between drug and co-former [7]. As a result, the US Food and Drug Administration (FDA) published guidance in 2013 which stated that co-crystals are not a new entity as such but should instead be treated as a "drug product intermediate" [8]. Attempts at co-crystal formation are performed with drugs within the second or fourth group in the Biopharmaceutical Classification System (BSC), with drugs characterised by poor solubility in water, a quality which, together with dissolution rate, is the key parameter as regards the bioavailability of drugs [6]. Many studies have shown that co-crystals improve the solubility in water and dissolution rates of parent compounds [9-11], and moreover, co-crystals can affect other physico-chemical properties of the same drug. In the 
literature can be found a description of an increase in physical and chemical stability or improvement in mechanical properties [12-14].

Screening and preparation of co-crystals can be achieved either by solid-state or solution-based methods [4, 12, 14-16]. The former consists of grinding methods without (neat grinding approach) or with the addition of a small amount of solvent (solvent-assisted grinding approach) [15]. In these methods, two substances are ground together with mortar and pestle or by mechanical grinders. Solvent evaporation, slurry conversion and cooling crystallisation are examples of traditional solutionbased methods [12, 14]. The recent literature provides various modifications of solution-based methods such as approaches using supercritical fluid technology [17] or an ultrasound-assisted solution process [13]. As solid-state and solution-based methods have some drawbacks [15], attempts at co-crystal formation are usually conducted using both methods together in order to obtain co-crystals with the most suitable properties, at least in the opinion of the researchers.

Regardless of the above methods, recent years have witnessed the emergence of reports about the application of differential scanning calorimetry (DSC) as a simple and rapid approach towards co-crystal screening [18-20]. This approach is based on the heating, by a DSC device, of binary physical mixtures of drug and co-former. Detailed interpretation of the DSC scans obtained may serve as a rapid screening approach that allows co-crystal detection. This is based on the assumption that the melting point of co-crystal differs from that of output components, since in more than $50 \%$ of cases co-crystals showed a melting point lower than that of both drug and co-former [21]. Generally, when the physical mixture capable of co-crystal formation is heated, the following thermal effects should occur on the DSC scan: an exothermic peak associated with co-crystal formation directly after an endothermic peak, together with another endothermic effect recorded at a higher temperature than the first peaks and connected with co-crystal melting, or several endothermic peaks in temperatures different to the melting points of the pure components and associated with metastable eutectic melting, eutectic melting and co-crystal melting [18, 19, 22]. Furthermore, the possibility of co-crystal screening using the DSC method has also been described, where co-crystals could be prepared at different molar ratios [23]. In this case, a DSC scan of the physical mixture shows two exothermic and three endothermic peaks. In contrast, when a physical mixture incapable of co-crystal formation is heated, only a single endothermic peak associated with eutectic melting appears on the DSC scan.

Taking all the above into consideration, the aim of this research was to verify to what extent the DSC method can be used as a rapid screening tool for detecting the cocrystallisation process in binary physical mixtures of benzodiazepines and co-formers. Benzodiazepines are psychoactive drugs with a wide range of medical applications, e.g. in treating anxiety, epileptic seizures or problems with insomnia, but are also applied as sedative drugs in premedication prior to medical or dental procedures [24]. Benzodiazepines, however, are classified as drugs very slightly or practically insoluble in water and with a low dissolution rate, a fact which has immediate effects on their poor bioavailability. As this may adversely affect efficiency or increase the occurrence of side effects in pharmacotherapy [25], many methods including physical or chemical modification of a drug have been applied to enhance the water solubility of benzodiazepines, such as particle size reduction, solubilisation by surfactants or the formation of salts and complexes [26, 27]. Because of the recent surge in interest in co-crystal formation as a promising additional way to enhance the water solubility of drugs, the preparation of co-crystals of benzodiazepines with selected co-formers, such as low molecular organic acids and other chemically neutral compounds, has become even more crucial. The co-formers chosen for this study are readily water soluble, non-toxic and with no negative effects on the human body. Nevertheless, to the best of the author's knowledge, there have been no reports on cocrystals of benzodiazepines.

\section{Experimental}

\section{Chemicals}

Benzodiazepines-chlordiazepoxide, clonazepam, diazepam, estazolam, lorazepam, lormetazepam, oxazepam and temazepam-were supplied courtesy of Polfa Tarchomin (Warsaw, Poland). The co-formers used in the study were acquired from Sigma-Aldrich (St. Louis, USA)- $p$ aminobenzoic acid, caffeine, fumaric acid, glutaric acid, nicotinamide and succinic acid; Acros Organics (New Jersey, USA) — citric acid and saccharin; and POCH (Gliwice, Poland)_urea. Both benzodiazepines and co-formers were characterised by a purity level above $98 \%$ and were used without further purification.

\section{Sample preparation}

Binary physical mixtures of benzodiazepines and coformers at 1:1 molar ratios were prepared in dust extraction hood by the gentle mixing of both ingredients in an agate mortar using plastic spatula. To achieve complete homogenisation, benzodiazepines and co-formers were 
thoroughly mixed with spatula over $8 \mathrm{~min}$. The samples obtained were then analysed by the DSC method.

To prepare the physical mixture, fixed amount of benzodiazepine equal to $0.05 \mathrm{~mol}$ was mixed with $0.05 \mathrm{~mol}$ of co-former that amount in milligrams varied depending on molar mass of co-former used. Both ingredients were weighed using analytical balance model XA 105 Dual Range (Mettler Toledo, Schwerzenbach, Switzerland).

\section{Differential scanning calorimetry}

A heat-flux DSC instrument model $822^{\mathrm{e}}$ (Mettler Toledo, Schwerzenbach, Switzerland) was used to obtain scans of benzodiazepines, co-formers and their binary mixtures. The device was connected with STARe software. Samples of 4.00-4.05 mg were placed into flat-bottomed aluminium pans, which were then sealed with perforated lids. The samples were measured over a range from 25 to $300{ }^{\circ} \mathrm{C}$, except for mixtures with fumaric acid, for which measurements were taken from 25 to $350{ }^{\circ} \mathrm{C}$. All measurements were taken under nitrogen flow (flux rate of $70 \mathrm{~mL} \mathrm{~min}{ }^{-1}$ ) at a heating rate $10{ }^{\circ} \mathrm{C} \mathrm{min}^{-1}$. DCS instrument was calibrated using indium and zinc as standards with regard to temperature and enthalpy.<smiles>[R6]C1=CC2=C([R5])N([R3])C([R])=C([R6])N2C=C1</smiles>

Fig. 1 1,4-Benzodiazepine ring-core structure of benzodiazepines. $\mathrm{R}_{1}, \mathrm{R}_{2}, \mathrm{R}_{3}, \mathrm{R}_{4}, \mathrm{R}_{5}$ and $\mathrm{R}_{6}$ denote different side groups attached to the core structure

\section{Results and discussion}

In this research, a total set of 72 binary physical mixtures composed of eight benzodiazepines (diazepam, temazepam, oxazepam, lormetazepam, lorazepam, clonazepam, estazolam and chlordiazepoxide) and nine co-formers (succinic, glutaric, fumaric, citric and $p$-aminobenzoic acids, nicotinamide, saccharin, urea and caffeine) mixed at 1:1 molar ratios were investigated using the DSC method. This method allows the more subtle differences in heat flow between the sample under study and the reference to be measured; thus, it may also be used to illustrate the physical changes (phase transitions) that occur in the mixtures of benzodiazepines with co-formers when heated under non-isothermal conditions. Melting (endothermic process) and crystallisation (exothermic process) are the most crucial phase transitions, as knowledge of these transitions is fundamental for the detection of potential cocrystals. For this reason, each change in the DSC scan of physical mixture in comparison with those of benzodiazepine and co-former alone can be interpreted as proof of co-crystal formation. Consequently, the DSC method can serve as a reliable screening method for co-crystallisation.

\section{Benzodiazepines and co-formers}

The core structure of benzodiazepines, the 1,4-benzodiazepine ring, is shown in Fig. 1, while the substituents attached to this structure are given in Table 1. The similar chemical structure of benzodiazepines is an effect of the connection of benzene and diazepine rings [24, 25]. As benzodiazepines have different side groups attached to the core structure, the drugs used in this study were classified as 5-phenyl-1,4-benzodiazepin-2-one derivatives including 1-methyl-substituted (diazepam), 3-hydroxy-substituted (temazepam, oxazepam, lormetazepam, lorazepam) and

Table 1 Substituents $R_{1}-R_{6}$ at the core structure of benzodiazepines-1,4-benzodiazepine ring

\begin{tabular}{|c|c|c|c|c|c|c|}
\hline Benzodiazepines & $\mathrm{R}_{1}$ & $\mathrm{R}_{2}$ & $\mathrm{R}_{3}$ & $\mathrm{R}_{4}$ & $\mathrm{R}_{5}$ & $\mathrm{R}_{6}$ \\
\hline Diazepam & $-\mathrm{CH}_{3}$ & $=\mathrm{O}$ & $-\mathrm{H}$ & - & $-\mathrm{C}_{6} \mathrm{H}_{5}$ & $-\mathrm{Cl}$ \\
\hline Temazepam & $-\mathrm{CH}_{3}$ & $=\mathrm{O}$ & $-\mathrm{OH}$ & - & $-\mathrm{C}_{6} \mathrm{H}_{5}$ & $-\mathrm{Cl}$ \\
\hline Oxazepam & $-\mathrm{H}$ & $=\mathrm{O}$ & $-\mathrm{OH}$ & - & $-\mathrm{C}_{6} \mathrm{H}_{5}$ & $-\mathrm{Cl}$ \\
\hline Lormetazepam & $-\mathrm{CH}_{3}$ & $=\mathrm{O}$ & $-\mathrm{OH}$ & - & $-\mathrm{C}_{6} \mathrm{H}_{4} \mathrm{Cl}$ & $-\mathrm{Cl}$ \\
\hline Lorazepam & $-\mathrm{H}$ & $=\mathrm{O}$ & $-\mathrm{OH}$ & - & $-\mathrm{C}_{6} \mathrm{H}_{4} \mathrm{Cl}$ & $-\mathrm{Cl}$ \\
\hline Clonazepam & $-\mathrm{H}$ & $=\mathrm{O}$ & $-\mathrm{H}$ & - & $-\mathrm{C}_{6} \mathrm{H}_{4} \mathrm{Cl}$ & $-\mathrm{NO}_{2}$ \\
\hline Estazolam & & & $-\mathrm{H}$ & - & $-\mathrm{C}_{6} \mathrm{H}_{5}$ & $-\mathrm{Cl}$ \\
\hline Chlordiazepoxide & - & $-\mathrm{NHCH}_{3}$ & $-\mathrm{H}$ & oxide & $-\mathrm{C}_{6} \mathrm{H}_{5}$ & $-\mathrm{Cl}$ \\
\hline
\end{tabular}

$\mathrm{R}_{1}, \mathrm{R}_{2}, \mathrm{R}_{3}, \mathrm{R}_{4}, \mathrm{R}_{5}$ and $\mathrm{R}_{6}$ denote different side groups attached to the core structure 
Table 2 General characteristics of benzodiazepines including temperatures of peaks $\left(T_{\text {peak }}\right)$ and enthalpies of transitions $(\Delta H)$ acquired from DSC scans

\begin{tabular}{|c|c|c|c|c|c|c|c|}
\hline Benzodiazepines & $\begin{array}{l}\text { Pharmacological } \\
\text { activity }\end{array}$ & $\begin{array}{l}\text { Chemical } \\
\text { formula } \\
\text { Molecular mass }\end{array}$ & $\begin{array}{l}\text { Crystalline } \\
\text { form }\end{array}$ & Solubility in water & $\begin{array}{l}T_{\text {peak }} / \\
{ }^{\circ} \mathrm{C}\end{array}$ & $\begin{array}{l}\Delta H / \\
\mathrm{J} \mathrm{g}^{-1}\end{array}$ & Literature data $/{ }^{\circ} \mathrm{C}$ \\
\hline Diazepam & $\begin{array}{l}\text { Anxiety disorder, } \\
\text { status epilepticus }\end{array}$ & $\begin{array}{l}\mathrm{C}_{16} \mathrm{H}_{13} \mathrm{ClN}_{2} \mathrm{O} \\
284.74\end{array}$ & $\begin{array}{l}\text { White or almost } \\
\text { white powder }\end{array}$ & $\begin{array}{l}\text { Very slightly } \\
\text { soluble }\end{array}$ & $\begin{array}{l}132.97^{\mathrm{a}} \\
287.09^{\mathrm{b}}\end{array}$ & $\begin{array}{l}89.24 \\
79.30\end{array}$ & $130.3[29]$ \\
\hline Temazepam & Insomnia & $\begin{array}{l}\mathrm{C}_{16} \mathrm{H}_{13} \mathrm{ClN}_{2} \mathrm{O}_{2} \\
300.74\end{array}$ & $\begin{array}{l}\text { White or almost } \\
\text { white powder }\end{array}$ & $\begin{array}{r}\text { Practically } \\
\text { insoluble }\end{array}$ & $\begin{array}{l}160.71^{\mathrm{a}} \\
244.04^{\mathrm{b}}\end{array}$ & $\begin{array}{l}94.71 \\
23.23\end{array}$ & $158.5[29]$ \\
\hline Oxazepam & Anxiety disorder & $\begin{array}{l}\mathrm{C}_{15} \mathrm{H}_{11} \mathrm{ClN}_{2} \mathrm{O}_{2} \\
286.71\end{array}$ & $\begin{array}{l}\text { White or almost } \\
\text { white powder }\end{array}$ & $\begin{array}{r}\text { Practically } \\
\text { insoluble }\end{array}$ & $\begin{array}{l}211.09^{\mathrm{a}} \\
304.08^{\mathrm{b}}\end{array}$ & $\begin{array}{l}299.85 \\
203.08\end{array}$ & $205.3 \pm 0.05[30]$ \\
\hline Lormetazepam & Insomnia & $\begin{array}{l}\mathrm{C}_{16} \mathrm{H}_{12} \mathrm{Cl}_{2} \mathrm{~N}_{2} \mathrm{O}_{2} \\
335.18\end{array}$ & White powder & Virtually insoluble & $\begin{array}{l}211.04^{\mathrm{a}} \\
247.68^{\mathrm{b}}\end{array}$ & $\begin{array}{l}104.58 \\
79.45\end{array}$ & $210.35 \pm 0.12[31]$ \\
\hline Lorazepam & $\begin{array}{l}\text { Anxiety disorder, } \\
\text { preanaesthetic } \\
\text { medication }\end{array}$ & $\begin{array}{l}\mathrm{C}_{15} \mathrm{H}_{10} \mathrm{Cl}_{2} \mathrm{~N}_{2} \mathrm{O}_{2} \\
321.20\end{array}$ & $\begin{array}{l}\text { White or almost } \\
\text { white powder }\end{array}$ & $\begin{array}{r}\text { Practically } \\
\text { insoluble }\end{array}$ & $183.21^{\mathrm{a}}$ & 248.36 & $182.6[32]$ \\
\hline Clonazepam & Seizure disorder & $\begin{array}{l}\mathrm{C}_{15} \mathrm{H}_{10} \mathrm{ClN}_{3} \mathrm{O}_{3} \\
315.71\end{array}$ & $\begin{array}{l}\text { Slightly } \\
\text { yellowish } \\
\text { powder }\end{array}$ & $\begin{array}{r}\text { Practically } \\
\text { insoluble }\end{array}$ & $\begin{array}{l}239.35^{\mathrm{a}} \\
289.16^{\mathrm{b}}\end{array}$ & $\begin{array}{l}112.46 \\
168.46\end{array}$ & $235.35[31]$ \\
\hline Estazolam & Insomnia & $\begin{array}{l}\mathrm{C}_{16} \mathrm{H}_{11} \mathrm{ClN}_{4} \\
294.74\end{array}$ & White powder & $\begin{array}{r}\text { Practically } \\
\text { insoluble }\end{array}$ & $231.10^{\mathrm{a}}$ & 100.42 & - \\
\hline Chlordiazepoxide & $\begin{array}{l}\text { Anxiety disorder, } \\
\text { management of } \\
\text { alcohol withdrawal }\end{array}$ & $\begin{array}{l}\mathrm{C}_{16} \mathrm{H}_{14} \mathrm{ClN}_{3} \mathrm{O} \\
299.75\end{array}$ & $\begin{array}{l}\text { Almost white or } \\
\text { pale yellow } \\
\text { powder }\end{array}$ & $\begin{array}{r}\text { Practically } \\
\text { insoluble }\end{array}$ & $\begin{array}{l}243.51^{\mathrm{a}} \\
286.16^{\mathrm{b}}\end{array}$ & $\begin{array}{l}108.91 \\
458.90\end{array}$ & - \\
\hline
\end{tabular}

Sign of the DSC peaks: a, endothermic; b, exothermic

7-nitro-substituted (clonazepam); derivative includes the addition of fused triazolo ring at the 1,2-position (estazolam); and N4-oxide 2-methylamine derivative (chlordiazepoxide). It is worth noting that benzodiazepines also have functional groups, i.e. hydroxyl $(-\mathrm{OH})$ and carbonyl $(\mathrm{C}=\mathrm{O})$, which are capable of forming intermolecular hydrogen bonds with co-formers.

Inspection of the physico-chemical data given in Table 2 reveals that molecular masses of benzodiazepines are located in a very narrow range between 284.74 (diazepam) and 335.18 (lormetazepam). The drugs in question are white or pale yellow crystalline powders, very slightly soluble in water (from 1000 to 10,000 parts of solvent required for one part of solute) or practically insoluble ( $>10,000$ parts of solvent required for one part of solute) [28]. This merely emphasises the importance of seeking new physical or chemical ways of modifying the water solubility of benzodiazepines, for example by co-crystallisation, to improve their bioavailability. Furthermore, analysis of melting points reveals that excluding diazepam, temazepam and lorazepam, the remaining benzodiazepines melt at high temperatures in excess of $200{ }^{\circ} \mathrm{C}$. This is in turn reflected in the high, narrow and sharp-ended DSC endothermic peaks, followed by exothermic peaks due to the decomposition of melted drugs. No exothermic peaks were observed in the case of lorazepam or estazolam. The DSC data obtained are consistent with those found in the literature [29-32], with, however, insignificant differences due to experimental conditions, such as heating rate or gas atmosphere.

Nine co-formers were used as the second component of potential benzodiazepine co-crystals-succinic, glutaric, fumaric, citric and $p$-aminobenzoic acids, nicotinamide, saccharin, urea and caffeine. Their structural formulas are illustrated in Fig. 2 and their primary physico-chemical properties in Table 3. The first fundamental condition in choosing organic compounds as co-formers is that they fulfil the status of generally recognized as safe (GRAS) [33], confirmation that the co-formers are not toxic and have no adverse effects on the human body. The second condition in the choice of co-former is the existence of functional groups that in their chemical structure are able to create so-called supramolecular synthons with molecules of psychoactive drugs which leads to the formation of intermolecular hydrogen bonds [33]. The carboxylic $(-\mathrm{COOH})$, amino $\left(-\mathrm{NH}_{2}\right)$, carbonyl $(\mathrm{C}=\mathrm{O})$ and hydroxyl $(-\mathrm{OH})$ groups are the most desirable in this respect (Fig. 2).

Table 3 shows that the co-formers used in this study are white or colourless crystalline powders, very soluble in water, over the range of $3.3 \mathrm{~g} \mathrm{~L}^{-1}$ (saccharin) to 
Fig. 2 Structural formulas of co-formers: a succinic acid, b glutaric acid, c fumaric acid, d citric acid, e $p$-aminobenzoic acid, $\mathbf{f}$ nicotinamide, g saccharin, $\mathbf{h}$ urea, $\mathbf{i}$ caffeine<smiles>O=C(O)CCC(=O)O</smiles>

d<smiles>O=C(O)CC(O)(CC(=O)O)C(=O)O</smiles>

g<smiles>O=C1NS(=O)(=O)c2ccccc21</smiles>

b<smiles>O=C(O)CCCC(=O)O</smiles>

e<smiles>Nc1ccc(C(=O)O)cc1</smiles>

h<smiles>NC(N)=O</smiles><smiles>C=C(C=CC(=O)O)C(=O)O</smiles>

f<smiles>NC(=O)c1cccnc1</smiles>

i<smiles>Cn1c(=O)c2c(ncn2C)n(C)c1=O</smiles>

Table 3 General characteristics of co-formers including temperatures of peaks $\left(T_{\text {peak }}\right)$ and enthalpies of transitions $(\Delta H)$ acquired from DSC scans

\begin{tabular}{|c|c|c|c|c|c|c|}
\hline Co-formers & $\begin{array}{l}\text { Chemical formula } \\
\text { Molecular mass }\end{array}$ & Crystalline form & $\begin{array}{l}\text { Solubility in } \\
\text { water/g } \mathrm{L}^{-1}\end{array}$ & $T_{\text {peak }} /{ }^{\circ} \mathrm{C}$ & $\Delta H / \mathrm{J} \mathrm{g}^{-1}$ & $\begin{array}{l}\text { Literature } \\
\text { data } /{ }^{\circ} \mathrm{C}\end{array}$ \\
\hline Succinic acid & $\begin{array}{l}\mathrm{C}_{4} \mathrm{H}_{6} \mathrm{O}_{4} \\
118.1\end{array}$ & White powder ${ }^{c}$ & $83^{\mathrm{c}}$ & $188.29^{\mathrm{a}}$ & 270.91 & $184.0[34]$ \\
\hline Glutaric acid & $\begin{array}{l}\mathrm{C}_{5} \mathrm{H}_{8} \mathrm{O}_{4} \\
132.1\end{array}$ & White or almost white powder ${ }^{c}$ & $430^{\mathrm{c}}$ & $\begin{array}{l}76.66^{\mathrm{a}} \\
98.25^{\mathrm{a}}\end{array}$ & $\begin{array}{l}19.69 \\
168.48\end{array}$ & $97.5[35]$ \\
\hline Fumaric acid & $\begin{array}{l}\mathrm{C}_{4} \mathrm{H}_{4} \mathrm{O}_{4} \\
116.1\end{array}$ & White powder ${ }^{c}$ & $7^{\mathrm{c}}$ & $\begin{array}{l}208.07^{\mathrm{a}} \\
295.81^{\mathrm{a}}\end{array}$ & $\begin{array}{l}13.89 \\
1015.65\end{array}$ & 287-302 [19] \\
\hline Citric acid & $\begin{array}{l}\mathrm{C}_{6} \mathrm{H}_{8} \mathrm{O}_{7} \\
192.1\end{array}$ & $\begin{array}{l}\text { White or almost white powder, colourless } \\
\text { crystals or granules }\end{array}$ & $750^{\mathrm{c}}$ & $\begin{array}{l}157.49^{\mathrm{a}} \\
217.55^{\mathrm{a}}\end{array}$ & $\begin{array}{l}206.05 \\
488.88\end{array}$ & $157.0[36]$ \\
\hline $\begin{array}{l}p \text {-aminobenzoic } \\
\text { acid }\end{array}$ & $\begin{array}{l}\mathrm{C}_{7} \mathrm{H}_{7} \mathrm{NO}_{2} \\
137.1\end{array}$ & Beige powder ${ }^{\mathrm{c}}$ & $5^{\mathrm{c}}$ & $\begin{array}{l}188.70^{\mathrm{a}} \\
244.58^{\mathrm{a}}\end{array}$ & $\begin{array}{l}177.59 \\
363.33\end{array}$ & $187.8[37]$ \\
\hline Nicotinamide & $\begin{array}{l}\mathrm{C}_{6} \mathrm{H}_{6} \mathrm{~N}_{2} \mathrm{O} \\
121.1\end{array}$ & $\begin{array}{l}\text { White or almost white powder or colourless } \\
\text { crystals }\end{array}$ & $\begin{array}{l}\text { Freely soluble } \\
\text { in water }\end{array}$ & $129.04^{\mathrm{a}}$ & 195.67 & $129.4[38]$ \\
\hline Saccharin & $\begin{array}{l}\mathrm{C}_{7} \mathrm{H}_{5} \mathrm{NO}_{3} \mathrm{~S} \\
183.2\end{array}$ & $\begin{array}{l}\text { White or almost white powder or colourless } \\
\text { crystals }\end{array}$ & $3.3^{\mathrm{c}}$ & $229.07^{\mathrm{a}}$ & 157.19 & $229.47[39]$ \\
\hline Urea & $\begin{array}{l}\mathrm{CH}_{4} \mathrm{~N}_{2} \mathrm{O} \\
60.1\end{array}$ & $\begin{array}{l}\text { White or almost white powder or transparent } \\
\text { crystals, slightly hygroscopic }\end{array}$ & $1080^{\mathrm{c}}$ & $\begin{array}{l}135.29^{\mathrm{a}} \\
245.29^{\mathrm{b}}\end{array}$ & $\begin{array}{l}226.90 \\
55.33\end{array}$ & $134.79[40]$ \\
\hline Caffeine & $\begin{array}{l}\mathrm{C}_{8} \mathrm{H}_{10} \mathrm{~N}_{4} \mathrm{O}_{2} \\
194.2\end{array}$ & $\begin{array}{l}\text { White or almost white powder or silky, white } \\
\text { or almost white, crystals }\end{array}$ & $18.7^{\mathrm{c}}$ & $\begin{array}{l}161.40^{\mathrm{a}} \\
236.23^{\mathrm{a}}\end{array}$ & $\begin{array}{l}15.38 \\
104.50\end{array}$ & $\sim 235[41]$ \\
\hline
\end{tabular}

Sign of the DSC peaks: a, endothermic; b, exothermic; c, material safety data sheet provided by producers

$1080 \mathrm{~g} \mathrm{~L}^{-1}$ (urea). Furthermore, they are characterised by a wide range of melting points, from $76.66{ }^{\circ} \mathrm{C}$ (glutaric acid) to $295.81{ }^{\circ} \mathrm{C}$ (fumaric acid). In the latter case, the DSC measurements for mixtures including this acid were taken up to $350{ }^{\circ} \mathrm{C}$, whereas remaining ingredients and mixtures were heated over a range $25-300{ }^{\circ} \mathrm{C}$. The melting of glutaric and fumaric acids, and caffeine is preceded by polymorphic transitions, which is confirmed by the small endothermic DSC peak. The melting of citric acid and urea is, in turn, followed by decomposition above $210{ }^{\circ} \mathrm{C}$, whereas melted $p$-aminobenzoic acid evaporates above $240{ }^{\circ} \mathrm{C}$. These data are consistent with those found in the literature [19, 34-41]. 
Table 4 Results of DSC analysis of the first group of benzodiazepines physical mixtures with co-formers

\begin{tabular}{llll}
\hline Physical mixtures & $\begin{array}{l}\text { Fumaric acid } \\
T_{\text {peak }} /{ }^{\circ} \mathrm{C}\end{array}$ & Citric acid & Nicotinamide \\
\hline Oxazepam & $165.35^{\mathrm{a}, \mathrm{s}}$ & $143.07^{\mathrm{a}, \mathrm{s}}$ & \\
& $206.28^{\mathrm{b}, \mathrm{s}}$ & $171.69^{\mathrm{b}, \mathrm{w}}$ & \\
& $255.50^{\mathrm{a}, \mathrm{s}}$ & $188.08^{\mathrm{a}, \mathrm{s}}$ & $129.94^{\mathrm{a}, \mathrm{w}}$ \\
Lorazepam & $151.80^{\mathrm{a}, \mathrm{s}}$ & & $133.67^{\mathrm{b}, \mathrm{w}}$ \\
& $217.58^{\mathrm{b}, \mathrm{s}}$ & & $180.00^{\mathrm{a}, \mathrm{s}}$ \\
\hline
\end{tabular}

Sign of the DSC peaks: a, endothermic; b, exothermic. Intensity of the DSC peaks: w, weak; m, medium; s, strong

Table 5 Results of DSC analysis of the second group of benzodiazepines physical mixtures with co-formers

\begin{tabular}{|c|c|c|c|c|c|}
\hline Physical mixtures & $\begin{array}{l}\text { Succinic acid } \\
T_{\text {peak }} /{ }^{\circ} \mathrm{C}\end{array}$ & Glutaric acid & Citric acid & p-Aminobenzoic acid & Urea \\
\hline Diazepam & $\begin{array}{l}124.41^{\mathrm{a}, \mathrm{m}} \\
176.71^{\mathrm{a}, \mathrm{m}} \\
226.34^{\mathrm{a}, \mathrm{m}}\end{array}$ & & $\begin{array}{l}122.17^{\mathrm{a}, \mathrm{m}} \\
148.33^{\mathrm{a}, \mathrm{w}} \\
181.58^{\mathrm{a}, \mathrm{s}}\end{array}$ & & \\
\hline Temazepam & & $\begin{array}{l}74.78^{\mathrm{a}, \mathrm{w}} \\
90.69^{\mathrm{a}, \mathrm{m}} \\
127.24^{\mathrm{a}, \mathrm{w}} \\
177.76^{\mathrm{b}, \mathrm{s}}\end{array}$ & & & $\begin{array}{l}133.88^{\mathrm{a}, \mathrm{m}} \\
144.28^{\mathrm{a}, \mathrm{m}} \\
209.19^{\mathrm{a}, \mathrm{s}}\end{array}$ \\
\hline Oxazepam & & $\begin{array}{l}75.42^{\mathrm{a}, \mathrm{w}} \\
98.53^{\mathrm{a}, \mathrm{m}} \\
123.03^{\mathrm{a}, \mathrm{s}} \\
187.60^{\mathrm{b}, \mathrm{s}}\end{array}$ & & $\begin{array}{l}154.17^{\mathrm{a}, \mathrm{m}} \\
182.65^{\mathrm{a}, \mathrm{s}} \\
241.03^{\mathrm{a}, \mathrm{w}} \\
245.30^{\mathrm{b}, \mathrm{s}}\end{array}$ & $\begin{array}{l}135.43^{\mathrm{a}, \mathrm{m}} \\
180.62^{\mathrm{a}, \mathrm{s}} \\
223.12^{\mathrm{a}, \mathrm{m}}\end{array}$ \\
\hline Lorazepam & & $\begin{array}{l}73.60^{\mathrm{a}, \mathrm{w}} \\
94.72^{\mathrm{a}, \mathrm{m}} \\
108.11^{\mathrm{a}, \mathrm{s}} \\
208.87^{\mathrm{b}, \mathrm{s}}\end{array}$ & $\begin{array}{l}134.96^{\mathrm{a}, \mathrm{s}} \\
152.51^{\mathrm{a}, \mathrm{m}} \\
193.52^{\mathrm{a}, \mathrm{s}}\end{array}$ & & \\
\hline Clonazepam & & & & & $\begin{array}{l}137.30^{\mathrm{a}, \mathrm{s}} \\
213.84^{\mathrm{a}, \mathrm{m}} \\
230.95^{\mathrm{a}, \mathrm{w}} \\
287.66^{\mathrm{b}, \mathrm{s}}\end{array}$ \\
\hline
\end{tabular}

Sign of the DSC peaks: a, endothermic; b, exothermic. Intensity of the DSC peaks: w, weak; m, medium; s, strong

\section{Rapid co-crystal screening}

The results of the DSC investigation of 72 binary physical mixtures of benzodiazepines with co-formers are given in Tables 4-7 and presented graphically in Figs. 3-8. Detailed inspection of these data shows that the mixtures under study could be divided into four groups consisting of $5,10,48$ and 9 mixtures, respectively, depending on the number of endothermic and exothermic effects detected on the DSC scans. Special attention has been paid to the appearance of new peaks due to the melting or crystallisation of co-crystals, or the disappearance of peaks due to melting of benzodiazepines and co-formers. This provides one hint to suggest the co-crystallisation of drug with coformer. Moreover, the shift in DSC peaks to lower or higher temperatures and the change in their intensities were also taken into consideration.

\section{The first group of mixtures}

As shown in Table 4, the first group of samples under study includes oxazepam mixtures with fumaric and citric acids, and lorazepam mixtures with fumaric acid, nicotinamide and saccharin. This group is characterised by the appearance of a new exothermic effect on the DSC scan between two endothermic peaks. In the case of three mixtures- 
Table 6 Results of DSC analysis of the third group of benzodiazepines physical mixtures with co-formers

\begin{tabular}{|c|c|c|c|c|c|c|c|c|c|}
\hline $\begin{array}{l}\text { Physical } \\
\text { mixtures }\end{array}$ & $\begin{array}{l}\text { Succinic } \\
\text { acid } \\
T_{\text {peak }} /{ }^{\circ} \mathrm{C}\end{array}$ & $\begin{array}{l}\text { Glutaric } \\
\text { acid }\end{array}$ & $\begin{array}{l}\text { Fumaric } \\
\text { acid }\end{array}$ & $\begin{array}{l}\text { Citric } \\
\text { acid }\end{array}$ & $\begin{array}{l}\text { p-Aminobenzoic } \\
\text { acid }\end{array}$ & Nicotinamide & Saccharin & Urea & Caffeine \\
\hline Diazepam & & $82.62^{\mathrm{a}, \mathrm{s}}$ & $\begin{array}{l}129.03^{\mathrm{a}, \mathrm{s}} \\
266.22^{\mathrm{b}, \mathrm{s}}\end{array}$ & & $112.24^{\mathrm{a}, \mathrm{s}}$ & $113.13^{\mathrm{a}, \mathrm{s}}$ & $121.31^{\mathrm{a}, \mathrm{s}}$ & $\begin{array}{l}129.21^{\mathrm{a}, \mathrm{m}} \\
133.90^{\mathrm{a}, \mathrm{w}}\end{array}$ & $125.19^{\mathrm{a}, \mathrm{m}}$ \\
\hline Temazepam & $\begin{array}{l}122.62^{\mathrm{a}, \mathrm{w}} \\
145.91^{\mathrm{a}, \mathrm{s}} \\
187.37^{\mathrm{b}, \mathrm{s}}\end{array}$ & & $\begin{array}{l}155.31^{\mathrm{a}, \mathrm{s}} \\
188.59^{\mathrm{b}, \mathrm{s}}\end{array}$ & $\begin{array}{l}136.57^{\mathrm{a}, \mathrm{s}} \\
169.11^{\mathrm{a}, \mathrm{s}}\end{array}$ & $126.00^{\mathrm{a}, \mathrm{s}}$ & $\begin{array}{l}114.76^{\mathrm{a}, \mathrm{s}} \\
135.56^{\mathrm{a}, \mathrm{w}} \\
223.70^{\mathrm{b}, \mathrm{m}}\end{array}$ & $\begin{array}{l}140.05^{\mathrm{a}, \mathrm{m}} \\
204.24^{\mathrm{b}, \mathrm{s}}\end{array}$ & & $\begin{array}{l}143.97^{\mathrm{a}, \mathrm{s}} \\
169.76^{\mathrm{a}, \mathrm{w}}\end{array}$ \\
\hline Oxazepam & $\begin{array}{l}156.10^{\mathrm{a}, \mathrm{s}} \\
197.52^{\mathrm{b}, \mathrm{s}}\end{array}$ & & & & & $\begin{array}{l}125.66^{\mathrm{a}, \mathrm{m}} \\
169.81^{\mathrm{a}, \mathrm{s}} \\
212.49^{\mathrm{b}, \mathrm{s}}\end{array}$ & $\begin{array}{l}153.79^{\mathrm{a}, \mathrm{s}} \\
171.93^{\mathrm{b}, \mathrm{s}}\end{array}$ & & $176.23^{\mathrm{a}, \mathrm{s}}$ \\
\hline Lormetazepam & $\begin{array}{l}168.04^{\mathrm{a}, \mathrm{w}} \\
185.65^{\mathrm{a}, \mathrm{w}} \\
200.74^{\mathrm{b}, \mathrm{m}}\end{array}$ & $\begin{array}{l}98.33^{\mathrm{a}, \mathrm{m}} \\
164.37^{\mathrm{a}, \mathrm{s}} \\
186.86^{\mathrm{b}, \mathrm{s}}\end{array}$ & $\begin{array}{l}183.43^{\mathrm{a}, \mathrm{s}} \\
198.95^{\mathrm{b}, \mathrm{s}}\end{array}$ & $150.59^{\mathrm{a}, \mathrm{s}}$ & $152.48^{\mathrm{a}, \mathrm{s}}$ & $\begin{array}{l}125.11^{\mathrm{a}, \mathrm{w}} \\
178.53^{\mathrm{a}, \mathrm{m}} \\
230.00^{\mathrm{b}, \mathrm{s}}\end{array}$ & $\begin{array}{l}167.81^{\mathrm{a}, \mathrm{s}} \\
202.24^{\mathrm{b}, \mathrm{s}}\end{array}$ & $\begin{array}{l}135.20^{\mathrm{a}, \mathrm{m}} \\
189.22^{\mathrm{a}, \mathrm{s}} \\
253.30^{\mathrm{b}, \mathrm{s}}\end{array}$ & $\begin{array}{l}162.12^{\mathrm{a}, \mathrm{w}} \\
180.87^{\mathrm{a}, \mathrm{s}} \\
250.20^{\mathrm{b}, \mathrm{m}}\end{array}$ \\
\hline Lorazepam & $\begin{array}{l}143.63^{\mathrm{a}, \mathrm{s}} \\
216.15^{\mathrm{a}, \mathrm{s}}\end{array}$ & & & & $153.34^{\mathrm{a}, \mathrm{s}}$ & & & $\begin{array}{l}133.42^{\mathrm{a}, \mathrm{s}} \\
232.28^{\mathrm{a}, \mathrm{s}}\end{array}$ & $\begin{array}{l}151.71^{\mathrm{a}, \mathrm{s}} \\
221.70^{\mathrm{a}, \mathrm{m}}\end{array}$ \\
\hline Clonazepam & $\begin{array}{l}179.45^{\mathrm{a}, \mathrm{m}} \\
208.01^{\mathrm{a}, \mathrm{w}} \\
283.12^{\mathrm{b}, \mathrm{s}}\end{array}$ & $\begin{array}{l}74.93^{\mathrm{a}, \mathrm{w}} \\
98.32^{\mathrm{a}, \mathrm{s}}\end{array}$ & $\begin{array}{l}231.17^{\mathrm{a}, \mathrm{s}} \\
288.55^{\mathrm{b}, \mathrm{s}}\end{array}$ & $\begin{array}{l}152.53^{\mathrm{a}, \mathrm{s}} \\
179.52^{\mathrm{a}, \mathrm{m}} \\
279.59^{\mathrm{b}, \mathrm{s}}\end{array}$ & $\begin{array}{l}161.70^{\mathrm{a}, \mathrm{m}} \\
199.72^{\mathrm{a}, \mathrm{w}} \\
274.40^{\mathrm{b}, \mathrm{s}}\end{array}$ & $\begin{array}{l}125.71^{\mathrm{a}, \mathrm{s}} \\
284.08^{\mathrm{b}, \mathrm{s}}\end{array}$ & $\begin{array}{l}190.90^{\mathrm{a}, \mathrm{m}} \\
262.69^{\mathrm{b}, \mathrm{s}}\end{array}$ & & $\begin{array}{l}159.96^{\mathrm{a}, \mathrm{w}} \\
190.07^{\mathrm{as}} \\
292.88^{\mathrm{b}, \mathrm{m}}\end{array}$ \\
\hline Estazolam & $136.78^{\mathrm{a}, \mathrm{m}}$ & $76.04^{\mathrm{a}, \mathrm{m}}$ & $219.40^{\mathrm{a}, \mathrm{s}}$ & $\begin{array}{l}146.04^{\mathrm{am}} \\
182.66^{\mathrm{a}, \mathrm{s}}\end{array}$ & $\begin{array}{l}132.13^{\mathrm{a}, \mathrm{m}} \\
185.76^{\mathrm{a}, \mathrm{w}}\end{array}$ & $122.84^{\mathrm{a}, \mathrm{s}}$ & $162.53^{\mathrm{a}, \mathrm{s}}$ & $\begin{array}{l}136.78^{\mathrm{a}, \mathrm{m}} \\
196.92^{\mathrm{a}, \mathrm{s}}\end{array}$ & $\begin{array}{l}160.30^{\mathrm{a}, \mathrm{w}} \\
186.58^{\mathrm{a}, \mathrm{s}}\end{array}$ \\
\hline
\end{tabular}

Sign of the DSC peaks: a, endothermic; b, exothermic. Intensity of the DSC peaks: w, weak; m, medium; s, strong

Table 7 Results of DSC analysis of the fourth group of benzodiazepines physical mixtures with co-formers

\begin{tabular}{|c|c|c|c|c|c|c|c|c|c|}
\hline $\begin{array}{l}\text { Physical } \\
\text { mixtures }\end{array}$ & $\begin{array}{l}\text { Succinic } \\
\text { acid } \\
T_{\text {peak }} /{ }^{\circ} \mathrm{C}\end{array}$ & $\begin{array}{l}\text { Glutaric } \\
\text { acid }\end{array}$ & $\begin{array}{l}\text { Fumaric } \\
\text { acid }\end{array}$ & $\begin{array}{l}\text { Citric } \\
\text { acid }\end{array}$ & $\begin{array}{l}\text { p-Aminobenzoic } \\
\text { acid }\end{array}$ & Nicotinamide & Saccharin & Urea & Caffeine \\
\hline \multirow[t]{4}{*}{ Chlordiazepoxide } & $161.13^{\mathrm{a}, \mathrm{s}}$ & $92.38^{\mathrm{a}, \mathrm{m}}$ & \multirow[t]{4}{*}{$204.96^{\mathrm{b}, \mathrm{s}}$} & $145.55^{\mathrm{a}, \mathrm{m}}$ & $154.13^{\mathrm{a}, \mathrm{s}}$ & $127.46^{\mathrm{a}, \mathrm{m}}$ & \multirow[t]{4}{*}{$205.86^{\mathrm{b}, \mathrm{s}}$} & $135.00^{\mathrm{a}, \mathrm{w}}$ & $160.34^{\mathrm{a}, \mathrm{w}}$ \\
\hline & $207.37^{\mathrm{b}, \mathrm{s}}$ & $205.95^{\mathrm{b}, \mathrm{s}}$ & & $208.70^{\mathrm{b}, \mathrm{s}}$ & $211.44^{\mathrm{b}, \mathrm{s}}$ & $272.21^{\mathrm{b}, \mathrm{s}}$ & & $247.64^{\mathrm{b}, \mathrm{s}}$ & $200.22^{\mathrm{a}, \mathrm{s}}$ \\
\hline & \multirow{2}{*}{\multicolumn{2}{|c|}{$244.07^{\mathrm{a}, \mathrm{s}}$}} & & & & & & & $234.47^{\mathrm{a}, \mathrm{s}}$ \\
\hline & & & & & & & & & $288.12^{\mathrm{b}, \mathrm{s}}$ \\
\hline
\end{tabular}

Sign of the DSC peaks: a, endothermic; b, exothermic. Intensity of the DSC peaks: w, weak; m, medium; s, strong

oxazepam with fumaric acid, and lorazepam with nicotinamide and saccharin-the exothermic peak occurs immediately after the first endothermic DSC effect, as illustrated in Fig. 3. The endothermic peaks appear at 129.94 and $180.00{ }^{\circ} \mathrm{C}$, and the new exothermic peak at $133.67{ }^{\circ} \mathrm{C}$ directly following the first endothermic effect (Table 4). The occurrence of a small exothermic peak immediately after the endothermic could suggest the possibility of co-crystal formation under mixture heating. Nevertheless, endothermic peak temperatures are close to the melting points of the individual ingredients (Tables 2, 3 ), which creates uncertainty over interpretation. However, it does not exclude the possibility of co-crystal formation characterised by peak temperature only slightly different to that of the melting point of lorazepam.

In the case of oxazepam mixture with fumaric acid and lorazepam with saccharin, peak temperatures are significantly different to those of the melting points of ingredients. This could suggest interaction between components and the formation of a new structure under heating, a situation presented in Fig. 4. The first endothermic effect appears at $165.35{ }^{\circ} \mathrm{C}$ and is shifted to a temperature (about $\sim 45{ }^{\circ} \mathrm{C}$, Table 4) lower than that of the melting point of oxazepam. The second endothermic peak appears at $255.50{ }^{\circ} \mathrm{C}$, between the melting points of oxazepam and fumaric acid. 


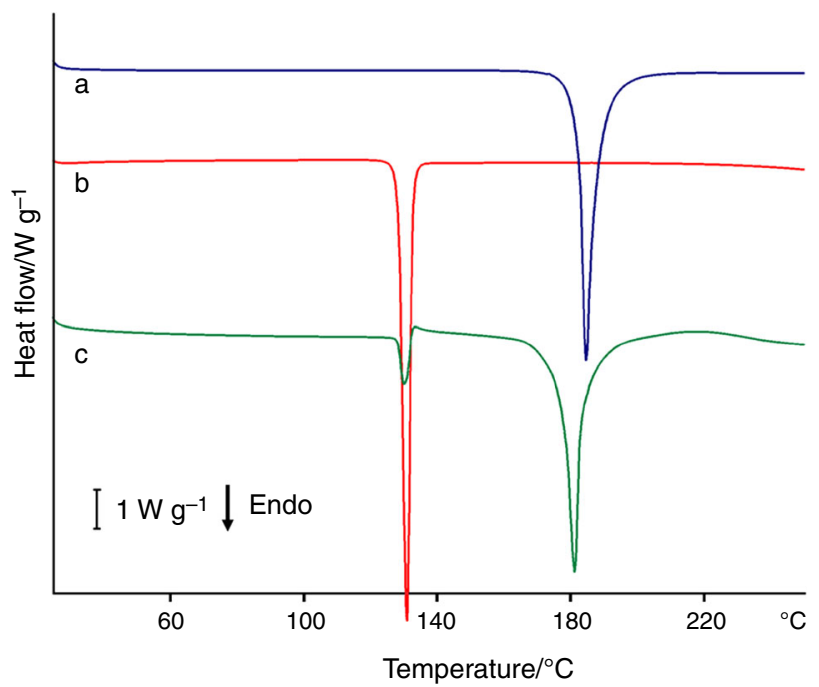

Fig. 3 DSC scans of: (a) lorazepam, (b) nicotinamide, (c) their physical mixture

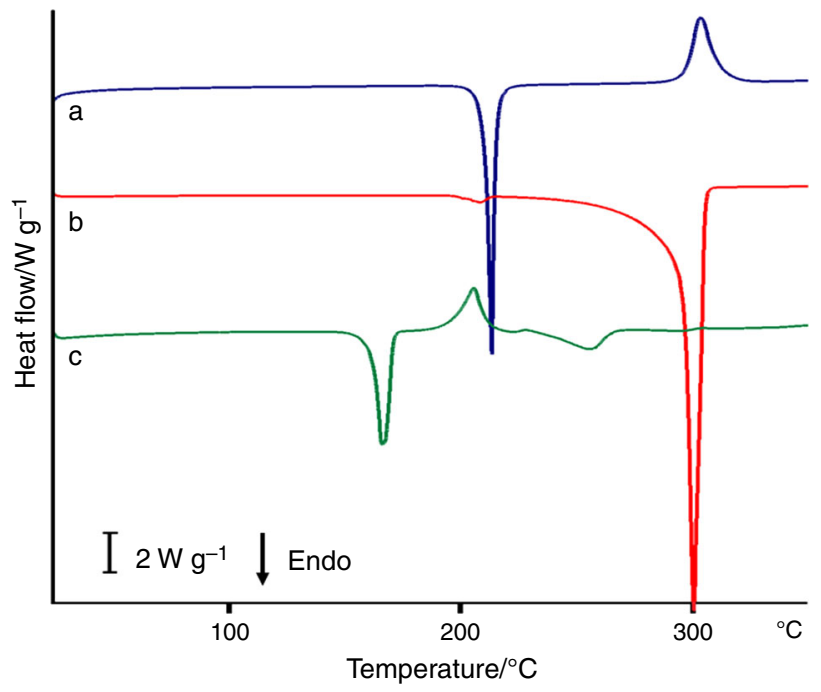

Fig. 4 DSC scans of: (a) oxazepam, (b) fumaric acid, (c) their physical mixture

In the case of oxazepam mixture with citric acid and lorazepam with fumaric acid, a new exothermic peak appears on the DSC scan at a temperature about $20^{\circ} \mathrm{C}$ higher in comparison with the first endothermic effect, which, in turn, is shifted to a lower temperature. This is untypical for mixtures capable of co-crystal formation. However, endothermic peaks (Table 4) do not overlap with the melting points of either ingredient (Tables 2,3), but are found at significantly lower temperatures than those of the melting points of ingredients. This could suggest interaction between components, possibly co-crystallisation.

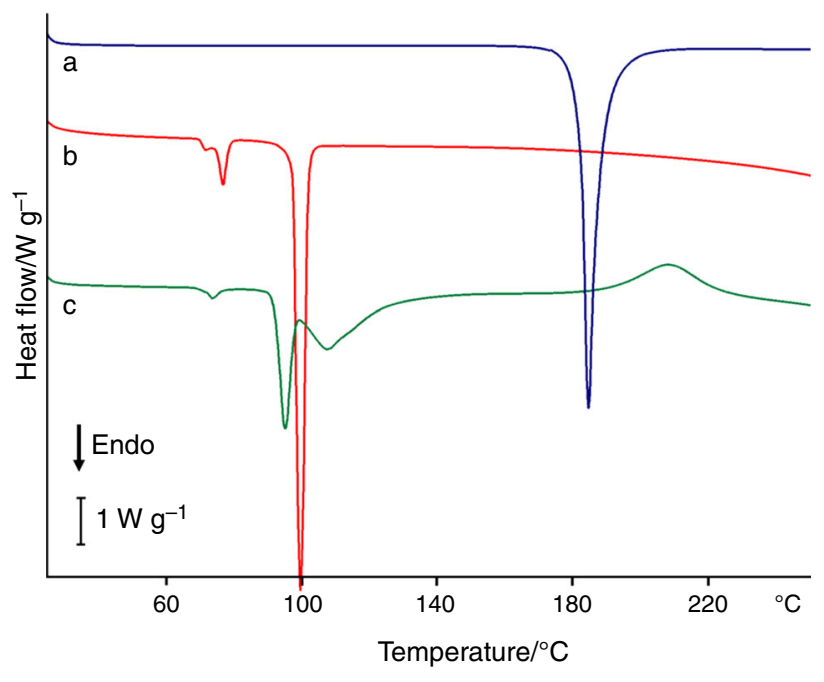

Fig. 5 DSC scans of: (a) lorazepam, (b) glutaric acid, (c) their physical mixture

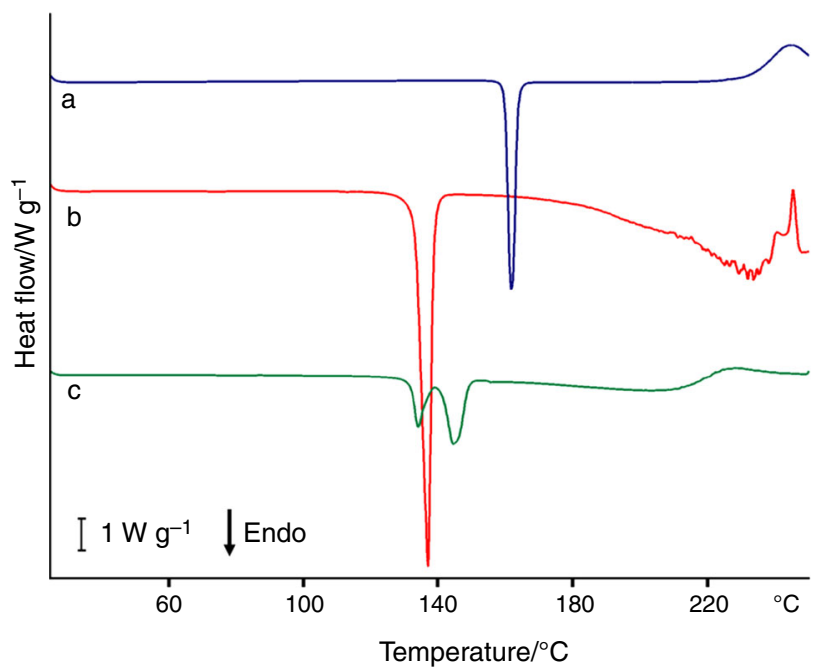

Fig. 6 DSC scans of: (a) temazepam, (b) urea, (c) their physical mixture

\section{The second group of mixtures}

Of 72 binary mixtures, ten (diazepam with succinic and citric acids, temazepam with glutaric acid and urea, oxazepam with glutaric and $p$-aminobenzoic acids and urea, lorazepam with glutaric and citric acids, and clonazepam with urea) form the second group (Table 5), distinguished by the occurrence at least three endothermic peaks on the DSC scan. These mixtures could be further divided into four subgroups, which are mixtures including glutaric acid, urea, diazepam and a final subgroup consisting of oxazepam mixture with $p$-aminobenzoic acid and lorazepam mixture with citric acid.

On the DSC scans of glutaric acid mixtures, the first peak (endothermic) appears at approximately $74{ }^{\circ} \mathrm{C}$ and 


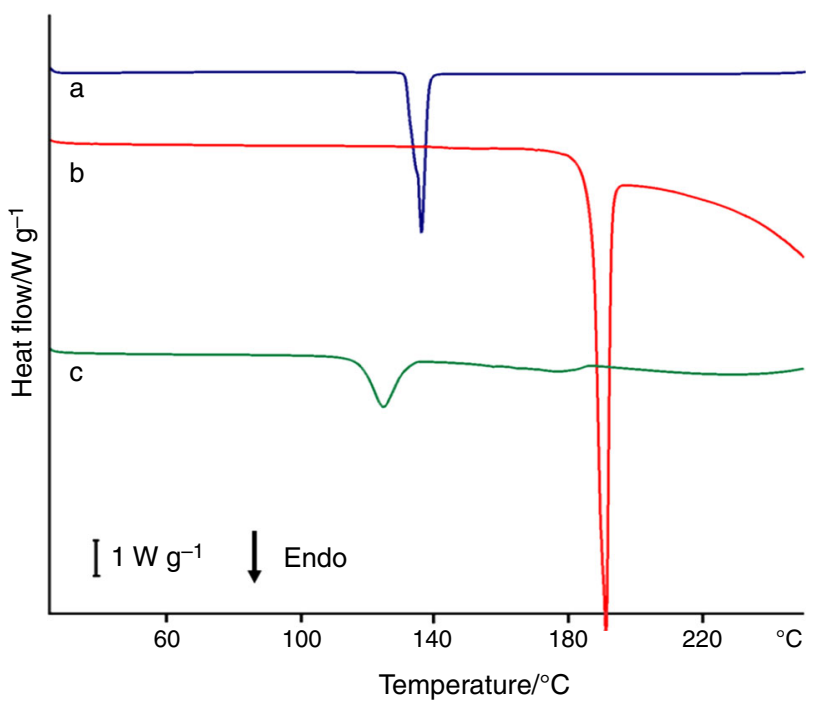

Fig. 7 DSC scans of: (a) diazepam, (b) succinic acid, (c) their physical mixture

could be associated with a transition from $\beta$-polymorph (the stable form at room temperature) to $\alpha$-polymorph. The second endothermic effect occurs over the range of 90-99 ${ }^{\circ} \mathrm{C}$ and could be assigned to the melting of the $\alpha$ polymorph [35]. This is illustrated in Fig. 5, which presents the DSC scan of lorazepam mixture with glutaric acid as an example. Insignificant differences in temperatures of the second peak in subsequent mixtures could be due to the influence of benzodiazepines on the melting of glutaric acid. The heats of fusion reflected by the second peak are similar for all glutaric acid mixtures (Table 5); however, the values are more than three times lower than the heat of fusion of glutaric acid (Table 3). The third peak occurs at 108.11, 123.03 and $127.24{ }^{\circ} \mathrm{C}$ for lorazepam, oxazepam and temazepam mixtures, respectively. This endothermic peak does not overlap with the peak solely due to the melting of benzodiazepine alone (Table 2). Rather it may suggest interaction between components, although additional and detailed investigation of this interaction is necessary. In all binary mixtures of glutaric acid, exothermic effects appear above $155^{\circ} \mathrm{C}$, probably due to sample decomposition.

In the case of mixtures with urea, the first endothermic effect is found at approximately $135{ }^{\circ} \mathrm{C}$ and could be associated with the melting of the co-former (Table 3 ). Figure 6 illustrates this through the example of a DSC scan of temazepam mixture with urea. There is an overlap with the first endothermic peak at $133.88^{\circ} \mathrm{C}$ due to the melting of urea; however, the heat of fusion reflected by this peak is more than eight times lower than that for co-former alone. At $144.28{ }^{\circ} \mathrm{C}$, the second endothermic peak appears, but the temperature differs from that for the melting of temazepam (Table 2). The last broad endothermic peak found on the DSC scan above $180{ }^{\circ} \mathrm{C}$ could not be associated with the melting point of either component, suggesting that interaction occurs under heating.

The third subgroup contains diazepam mixtures with succinic and citric acids. The DSC scans of these mixtures show three endothermic effects, reflected in Fig. 7 which presents the DSC scan of diazepam mixture with succinic acid. The first and second peaks are found at temperatures approximately $10{ }^{\circ} \mathrm{C}$ lower than that of the melting points of drug and both co-formers. The third broad endothermic peak occurs above $180{ }^{\circ} \mathrm{C}$. The situation described could imply interaction between components under heating or could be attributed to the influence of ingredients on their melting points. Again, additional study is required to clarify this issue.

The last subgroup consists of mixtures of oxazepam with $p$-aminobenzoic acid and lorazepam with citric acid. The first endothermic peak occurs at a temperature significantly lower than that for the melting of the constituent parts. The second endothermic peak, as with the first, is found at a lower temperature (about $6{ }^{\circ} \mathrm{C}$ ) than that for the melting points of benzodiazepine and co-former, and the last is detected at a temperature higher than that of melting points of oxazepam and lorazepam. In the case of oxazepam mixture with $p$-aminobenzoic acid, the DSC scan additionally revealed an exothermic peak immediately after the third endothermic peak due to sample decomposition.

\section{The third group of mixtures}

The third group of samples under study includes 48 mixtures given in Table 6. Their DSC scans illustrate those events in which no formation of co-crystals was confirmed. In those cases, one or two endothermic peaks are found. Furthermore, in the case of sparse mixtures, an additional exothermic peak was observed at higher temperatures due to sample decomposition. The situation in which single endothermic peak occurs on the DSC scan is shown in Fig. 8. The peak temperature at $152.48{ }^{\circ} \mathrm{C}$ for lormetazepam mixture with $p$-aminobenzoic acid is lower than that for melting of drug and co-former alone. A single endothermic effect is evidence that both components form an eutectic mixture, which precludes the possibility of obtaining co-crystals [18]. Above $280{ }^{\circ} \mathrm{C}$, a small exothermic effect appeared due to decomposition. On the other hand, two endothermic peaks are found in the case of estazolam mixture with $p$-aminobenzoic. A sharp endothermic peak at $132.13{ }^{\circ} \mathrm{C}$ on the DSC scan converting into a broad endothermic event. The temperatures of both peaks (Table 6) are lower than those for melting of estazolam and $p$-aminobenzoic acid (Tables 2,3). This also suggests the formation of an eutectic mixture, which again 


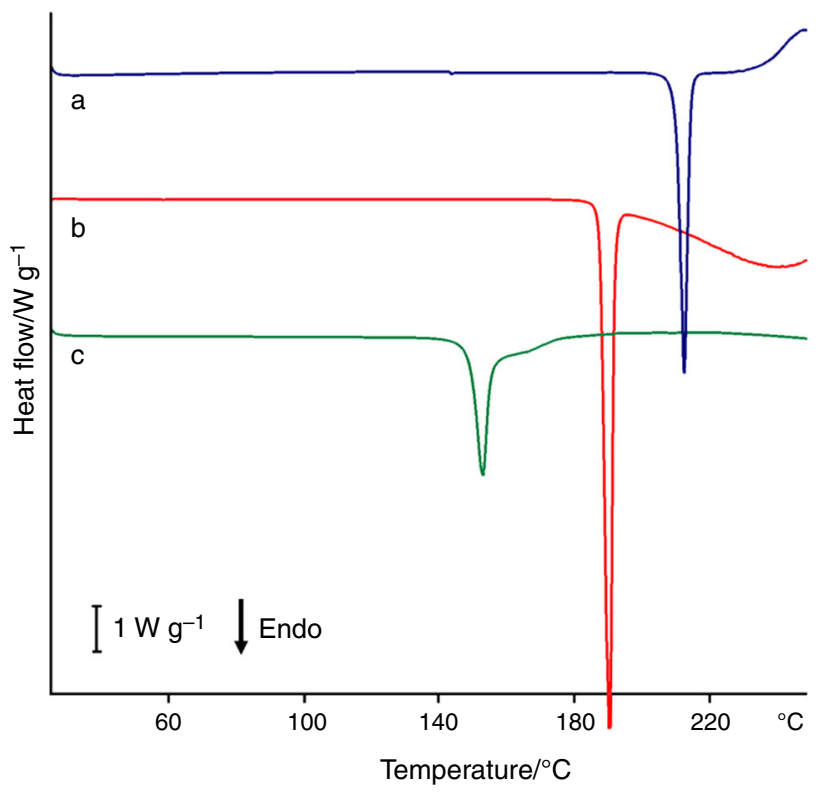

Fig. 8 DSC scans of: (a) lormetazepam, (b) p-aminobenzoic acid, (c) their physical mixture

implies the impossibility of co-crystal formation between drug and co-former.

\section{The fourth group of mixtures}

As shown in Table 7, the nine mixtures consisting of all chlordiazepoxide mixtures are gathered into the fourth group. This drug melts at $243.51{ }^{\circ} \mathrm{C}$ and then decomposes, which is reflected by two subsequent DSC peaks: endothermic and exothermic (Table 2). A detailed analysis of the DSC scans of chlordiazepoxide mixtures with coformers reveals that the predominant exothermic peaks due to decomposition hinder the retrieval of reliable data. For this reason, the DSC scans of mixtures with chlordiazepoxide cannot be correctly interpreted. This also demonstrates the dubious effectiveness of DSC as a screening method for co-crystal formation under the heating of binary mixtures, including ingredients melted with decomposition. This result is consistent with the previous study [22].

\section{Conclusions}

The study revealed that the DSC method can be successfully applied in order to detect a co-crystallisation process occurring in a pharmaceutical mixture when heated under non-isothermal conditions. The detailed interpretation of the DSC scans of 72 binary physical mixtures including benzodiazepines (very slightly or practically insoluble in water) and selected co-formers shows that the appearance of new endothermic or exothermic DSC peaks due to melting or crystallisation of newly obtained co-crystals, or the disappearance of DSC peaks due to the melting of benzodiazepines and co-formers, provides an argument that potential co-crystals can be found in the mixture under heating.

Based on the results obtained in this study, 15 mixtures including diazepam, temazepam, oxazepam, lorazepam and clonazepam were selected as capable of forming potential co-crystals with co-formers when heated. There are mixtures of oxazepam with glutaric, fumaric, citric and $p$ aminobenzoic acids and urea; lorazepam with glutaric, fumaric and citric acids, nicotinamide and saccharin; diazepam with succinic and citric acids; temazepam with glutaric acid and urea; and clonazepam with urea. Furthermore, oxazepam and lorazepam were identified as the benzodiazepines which most readily undergo co-crystallisation, each drug forming at least five co-crystals. With regard to co-formers, glutaric acid, citric acid and urea participated most frequently in the co-crystallisation process, each co-former forming at least three co-crystals. On the other hand, no mixture containing lormetazepam and estazolam formed co-crystals. A similar situation has also been observed in the case of caffeine. The DSC scans confirmed that no co-crystals were obtained in any of the caffeine mixtures.

To emphasise the importance of DSC as a rapid screening tool for the detection of co-crystallisation process in binary physical mixtures under heating, the next stage of research should include other instrumental methods to confirm the findings obtained in this study. The preliminary results outlined only indicate the direction of study to enhance benzodiazepine bioavailability.

Open Access This article is distributed under the terms of the Creative Commons Attribution 4.0 International License (http://creative commons.org/licenses/by/4.0/), which permits unrestricted use, distribution, and reproduction in any medium, provided you give appropriate credit to the original author(s) and the source, provide a link to the Creative Commons license, and indicate if changes were made.

\section{References}

1. Ledeti A, Vlase G, Vlase T, Circioban D, Dehelean C, Ledeti I, Suta LM. Binary adduct formation of desipramine with dicarboxylic acids. An instrumental study. J Therm Anal Calorim. 2016;. https://doi.org/10.1007/s10973-016-5967-8.

2. Mohammad KA, Rahim SA, Bakar MRA. Kinetics and nucleation mechanism of carbamazepine-saccharin co-crystals in ethanol solution. J Therm Anal Calorim. 2017; . https://doi.org/10. 1007/s10973-017-6483-1.

3. Lin H-L, Huang Y-T, Lin S-Y. Spectroscopic and thermal approaches to investigate the formation mechanism of piroxicamsaccharin co-crystal induces by liquid-assisted grinding or thermal stress. J Therm Anal Calorim. 2016;123:2345-56. 
4. Lin H-L, Wu T-K, Lin S-Y. Screening and characterization of cocrystal formation of metaxalone with short-chain dicarboxylic acids induced by solvent-assisted grinding approach. Thermochim Acta. 2014;575:313-21.

5. Alhalaweh A, George S, Basavoju S, Childs SL, Rizvi SAA, Velaga SP. Pharmaceutical cocrystals of nitrofurantoin: screening, characterization and crystal structure analysis. CrystEngComm. 2012;14:5078-88.

6. Haneef J, Chadha R. Drug-drug multicomponent solid forms: cocrystal, coamorphous and eutectic of three poorly soluble antihypertensive drugs using mechanochemical approach. AAPS PharmSciTech. 2017;18:2279-90.

7. Évora AOL, Castro RAE, Maria TMR, Silva MR, Ter Horst JH, Canotilho J, Eusebio MES. A thermodynamic based approach on the investigation of a diflunisal pharmaceutical co-crystal with improved intrinsic dissolution rate. Int J Pharm. 2014;466:68-75.

8. FDA. Guidance for industry. Regulatory classification of pharmaceutical co-crystals. 2013. p. 1-5. http://www.fda.gov/down loads/Drugs/Guidances/UCM281764.pdf. Accessed 29 Nov 2015.

9. Jung M-S, Kim J-S, Kim M-S, Alhalaweh A, Cho W, Hwang S-J, Velaga SP. Bioavailability of indomethacin-saccharin cocrystals. J Pharm Pharmacol. 2010;62:1560-8.

10. Huang Y, Zhang B, Gao Y, Zhang J, Shi L. Baicalein-nicotinamide cocrystal with enhanced solubility, dissolution, and oral bioavailability. J Pharm Sci. 2014;103:2330-7.

11. Shayanfar A, Asadpour-Zeynali K, Jouyban A. Solubility and dissolution rate of a carbamazepine-cinnamic acid cocrystal. J Mol Liq. 2013;187:171-6.

12. Rahman Z, Agarabi C, Zidan AS, Khan SR, Khan MA. Physicomechanical and stability evaluation of carbamazepine cocrystal with nicotinamide. AAPS PharmSciTech. 2011;12:693-704.

13. Aher S, Dhumal R, Mahadik K, Ketolainen J, Paradkar A. Effect of cocrystallization techniques on compressional properties of caffeine/oxalic acid 2:1 cocrystal. Pharm Dev Technol. 2013;18:55-60.

14. Trask AV, Motherwell WDS, Jones W. Physical stability enhancement of theophylline via cocrystallization. Int J Pharm. 2006;320:114-23.

15. Trask AV, Jones W. Crystal engineering of organic cocrystals by the solid-state grinding approach. Top Curr Chem. 2005;254:41-70.

16. Rahim SA, Hammond RB, Sheikh AY, Roberst KJ. A comparative assessment of the influence of different crystallization screening methodologies on the solid forms of carbamazepine cocrystals. CrystEngComm. 2013;15:3862-73.

17. Padrela L, Rodrigues MA, Velaga SP, Matos HA, de Azevedo EG. Formation of indomethacin-saccharin cocrystals using supercritical fluid technology. Eur J Pharm Sci. 2009;38:9-17.

18. Yamashita H, Hirakura Y, Yuda M, Teramura T, Terada K. Detection of cocrystal formation based on binary phase diagrams using thermal analysis. Pharm Res. 2013;30:70-80.

19. Yamashita H, Hirakura Y, Yuda M, Terada K. Coformer screening using thermal analysis based on binary phase diagrams. Pharm Res. 2014;31:1946-57.

20. Lu E, Rodriguez-Hornedo N, Suryanarayanan R. A rapid thermal method for cocrystal screening. CrystEngComm. 2008;10:665-8.

21. Schultheiss N, Newman A. Pharmaceutical cocrystals and their physicochemical properties. Cryst Growth Des. 2009;9:2950-67.

22. Manin AN, Voronim AP, Drozd KV, Manin NG, Bauer-Brandl A, Perlovich GL. Cocrystal screening of hydroxybenzamides with benzoic acid derivatives: a comparative study of thermal and solution-based methods. Eur J Pharm Sci. 2014;65:56-64.

23. Zhou Z, Chan HM, Sung HH-Y, Tong HHY, Zheng Y. Identification of new cocrystal systems with stoichiometric diversity of salicylic acid using thermal methods. Pharm Res. 2016;33:1030-9.
24. Mihic SJ, Harris RA. Hypnotics and sedatives. In: Brunton LL, Chabner BA, Knollmann BC, editors. Goodman \& Gilman's: the pharmacological basis of therapeutics. 12th ed. New York: McGraw-Hill; 2011. http://accessmedicine.mhmedical.com/con tent.aspx ?bookid=1613\&sectionid=1021. Accessed 26 July 2017.

25. Trevor AJ. Sedative-hypnotic drugs. In: Katzung BG, Trevor JA, editors. Basic \& clinical pharmacology. 13th ed. New York: McGrawHill; 2011. http://accessmedicine.mhmedical.com/content.aspx?boo kid=1193\&sectionid=6910676. Accessed 26 July 2017.

26. Shayesteh TH, Radmehr M, Khajavi F, Mahjub R. Application of chemometrics in determination of the acid dissociation constants (pKa) of several benzodiazepine derivatives as poorly soluble drugs in the presence of ionic surfactants. Eur J Pharm Sci. 2015;69:44-50.

27. Mennini N, Bragagni M, Maestrelli F, Mura P. Physico-chemical characterization in solution and in the solid state of clonazepam complexes with native and chemically-modified cyclodextrins. J Pharm Biomed Anal. 2014;89:142-9.

28. European Pharmacopoeia. 9th ed. 2017. http://online.edqm.eu/ EN/entry.htm. Accessed 26 July 2017.

29. Bibi S, Bremner DH, Macdougall-Heasman M, Reid R, Simpson K, Tough A, Waddell S, Stewart IJ, Matthews KH. A preliminary investigation to group disparate batches of licit and illicit diazepam tablets using differential scanning calorimetry. Anal Methods. 2015;7:8597-604.

30. Arias MJ, Moyano JR, Ginés JM. Study by DSC and HSM of the oxazepam PEG 6000 and oxazepam D-mannitol system: application to the preparation of solid dispersions. Thermochim Acta. 1998;321:33-41.

31. Van Den Mooter G, Van Den Brande J, Augustijns P, Kinget R. Glass forming properties of benzodiazepines and co-evaporate systems with poly(hydroxyethyl methacrylate). J Therm Anal. 1999;57:493-507.

32. Jug M, Bećirević-Laćan M. Development of a cyclodextrin-based nasal delivery system for lorazepam. Drug Dev Ind Pharm. 2008;34:817-26.

33. Qiao N, Li M, Schlindwein W, Malek N, Davies A, Trappitt G. Pharmaceutical cocrystals: an overview. Int $\mathrm{J}$ Pharm. 2014;419:1-11.

34. Claeys B, De Bruyn S, Hansen L, De Beer T, Remon JP, Vervaet C. Release characteristics of polyurethane tablets containing dicarboxylic acids as release modifiers-a case study with diprophylline. Int J Pharm. 2014;477:244-50.

35. Bruni G, Maietta M, Maggi L, Mustarelli P, Ferrara C, Berbenni V, Milanese C, Girella A, Marini A. Preparation and physicochemical characterization of acyclovir cocrystals with improved dissolution properties. J Pharm Sci. 2013;102:4079-86.

36. Hsu P-C, Lin H-Y, Wang S-L, Lin S-Y. Solid-state thermal behavior and stability studies of theophylline-citric acid cocrystals prepared by neat cogrinding or thermal treatment. J Solid State Chem. 2012;192:238-45.

37. Sarraguça MC, Paisana M, Pinto J, Lopes JA. Real-time monitoring of cocrystallization processes by solvent evaporation: a near infrared study. Eur J Pharm Sci. 2016;90:76-84.

38. Cuadra IA, Cabañas A, Cheda JAR, Martínez-Casado FJ, Pando C. Pharmaceutical cocrystals of the anti-inflammatory drug diflunisal and nicotinamide obtained using supercritical $\mathrm{CO}_{2}$ as an antisolvent. J CO2 Util. 2016;13:29-37.

39. Basavoju S, Boström D, Velaga SP. Indomethacin-saccharin cocrystal: design, synthesis and preliminary pharmaceutical characterization. Pharm Res. 2008;25:530-41.

40. Goud RN, Gangavaram S, Kuthuru S, Sharmistha P, Manjunatha SG. Novel furosemide cocrystals and selection of high solubility drugs forms. J Pharm Sci. 2012;101:664-80.

41. Pal S, Roopa BN, Abu K, Manjunath SG, Nambiar S. Thermal studies of furosemide-caffeine binary system that forms a cocrystal. J Therm Anal Calorim. 2014;115:2261-8. 